\title{
Crystal structure of 1-[2-[6-(4-methoxyphenyl)-3(2H)-pyridazinone- 2-yl]-acetyl]-4-(3-chlorophenyl)piperazine, $\mathrm{C}_{23} \mathrm{H}_{23} \mathrm{ClN}_{4} \mathrm{O}_{3}$
}

\author{
Ş. K. İngeç*,1, H. Soylu1, K. Engin'"I D. S. Dogruer ${ }^{111}$ and M. F. Şahin ${ }^{I I I}$ \\ I Gazi University, Gazi Education Faculty, Physics Department, 06500, Beşevler, Ankara, Turkey \\ II Hacettepe University, Department of Engineering Physics, 06532, Beytepe, Ankara, Turkey \\ III Gazi University, Faculty of Pharmacy, Department of Pharmaceutical Chemistry, Hipodrum 06330, Ankara, Turkey
}

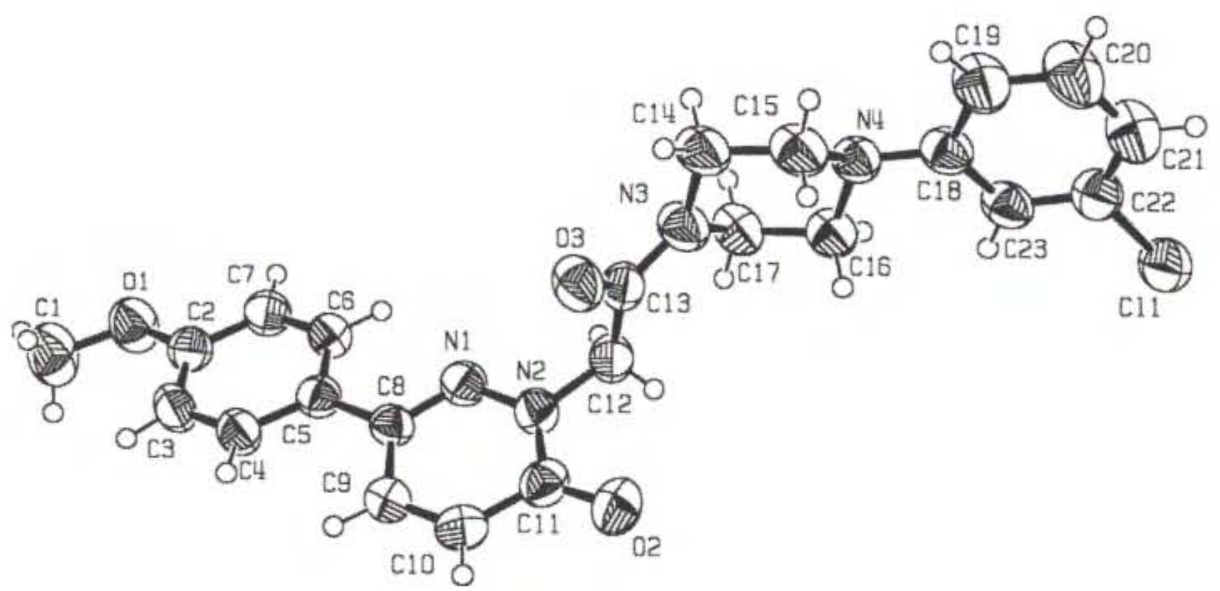

\begin{abstract}
$\mathrm{C}_{23} \mathrm{H}_{23} \mathrm{ClN}_{4} \mathrm{O}_{3}$, triclinic, $P \overline{1}$ (No. 2), $a=10.044(1) \AA$, $b=10.955(2) \AA, c=11.450(2) \AA, \alpha=69.161(9)^{\circ}$.

$\beta=70.761(5)^{\circ}, \gamma=72.90(1)^{\circ}, V=1089.1 \AA^{3}, Z=2$, $R_{\mathrm{gt}}(F)=0.053, w R_{\mathrm{ref}}\left(F^{2}\right)=0.157, T=293 \mathrm{~K}$.
\end{abstract}

\section{Source of material}

[6-(4-methoxyphenyl)-3(2H)-pyridazinone-2-yl]acetic acid $(0.01 \mathrm{~mol})$ in $40 \mathrm{ml}$ dichloromethane at $273 \mathrm{~K}$ (ice-bath) was treated with triethylamine $(3 \mathrm{ml})$ and $0.01 \mathrm{~mol}$ of ethyl chloroformate. After stirring the reaction mixture at $273 \mathrm{~K}$ for $15 \mathrm{~min}, 0.011 \mathrm{~mol}$ of 1-(3-chlorophenyl)piperazine was added to this solution. The final mixture was stirred at $273 \mathrm{~K}-298 \mathrm{~K}$ for $24 \mathrm{~h}$, evaporated to dryness, then treated with acetone. All solid materials thus obtained were washed with $1 \%$ solution of $\mathrm{NaOH}$, then with water, dried and crystallized from methanol.

\section{Discussion}

The torsion angles $\mathrm{C} 19-\mathrm{C} 18-\mathrm{N} 4-\mathrm{C} 15\left[42.7(6)^{\circ}\right]$ and C23-C18-N4-C15 $\left[-138.3(4)^{\circ}\right]$ define the position of the piperazine ring in the molecule. The piperazine ring may be described as a chair conformation with the N3 and N4 a toms deviàting by $-0.646(6) \AA$ and $0.601(6) \AA$, respectively, from the plane of the $\mathrm{C} 14, \mathrm{C} 15, \mathrm{C} 16$ and $\mathrm{C} 17$ atoms. The bond lengths and angles are comparable to those observed in related piperazine derivatives [1-3]. The pyridazine and two phenyl rings ( $\mathrm{C} 2-\mathrm{C} 7$ and $\mathrm{C} 18-\mathrm{C} 23$ ) are planar, the r.m.s. deviations from the mean planes are $0.009 \AA, 0.006 \AA$ and $0 . \overline{0} 08 \AA$. The dihedral angle between the pyridazine ring and piperazine ring is $82.5(1)^{\circ}$. The two phenyl rings (C2-C7 and $\mathrm{C} 18-\mathrm{C} 23)$ make dihedral angles of $88.2(1)^{\circ}$ to each other and angles of $72.9(1)^{\circ}$ and $21.9(2)^{\circ}$ to the piperazine ring, respectively. In the two phenyl rings the bond and angles are in good agreement with the expected value for aromatic rings [2]. The methoxy group is almost coplanar with the aromatic rings, as shown by torsion angles $174.7(4)^{\circ}$ for $\mathrm{C} 7-\mathrm{C} 2-\mathrm{O} 1-\mathrm{Cl}$. The $\mathrm{CO}$ $[1.407(6) \AA]$ and $\mathrm{C}=\mathrm{O}[1.233(4) \AA]$ distances are comparable with reported values $[4,5]$.

Table 1. Data collection and handling.

$\begin{array}{ll}\text { Crystal: } & \begin{array}{l}\text { colourless, prismatic, } \\ \text { size } 0.12 \times 0.18 \times 0.40 \mathrm{~mm}\end{array} \\ \text { Wavelength: } & \text { Cu } K_{\alpha} \text { radiation }(1.54184 \AA) \\ \mu: & 18.23 \mathrm{~cm}^{-1} \\ \text { Diffractometer, scan mode: } & \text { Enraf-Nonius CAD-4, } \omega / 2 \theta \\ 2 \theta_{\text {max }}: & 148.4^{\circ} \\ N(h k l)_{\text {measured }}, N(h k l)_{\text {unique: }} & 4441,4441 \\ \text { Criterion for } I_{\text {obs, }} N(h k l)_{\text {țt }}: & I_{\text {obs }}>2 \sigma\left(I_{\text {obs }}\right), 3274 \\ N(p a r a m)_{\text {refined: }} & 338 \\ \text { Programs: } & \text { SHELXS-97 [7], SHELXL-97 [8], } \\ & \text { ORTEPII [9] }\end{array}$

\footnotetext{
* Correspondence author (e-mail: singec@ sirius.gazi.edu.tr)
} 
Table 2. Atomic coordinates and displacement parameters (in $\AA^{2}$ ).

\begin{tabular}{llllll}
\hline Atom & Site & $x$ & $y$ & $z$ & $U_{\text {iso }}$ \\
\hline $\mathrm{H}(1 \mathrm{~A})$ & $2 i$ & 0.5148 & 1.6277 & 1.245 & 0.142 \\
$\mathrm{H}(1 \mathrm{~B})$ & $2 i$ & 0.6396 & 1.5475 & 1.313 & 0.142 \\
$\mathrm{H}(1 \mathrm{C})$ & $2 i$ & 0.4839 & 1.5182 & 1.3777 & 0.142 \\
$\mathrm{H}(3)$ & $2 i$ & $0.300(4)$ & $1.541(4)$ & $1.282(4)$ & $0.06(1)$ \\
$\mathrm{H}(4)$ & $2 i$ & $0.134(5)$ & $1.508(5)$ & $1.210(4)$ & $0.08(1)$ \\
$\mathrm{H}(6)$ & $2 i$ & 0.4457 & 1.2508 & 1.0378 & 0.067 \\
$\mathrm{H}(7)$ & $2 i$ & 0.6144 & 1.2983 & 1.0981 & 0.071 \\
$\mathrm{H}(9)$ & $2 i$ & $-0.024(4)$ & $1.439(4)$ & $1.179(4)$ & $0.049(9)$ \\
$\mathrm{H}(10)$ & $2 i$ & $-0.184(4)$ & $1.360(4)$ & $1.130(4)$ & $0.06(1)$ \\
$\mathrm{H}(12 \mathrm{~A})$ & $2 i$ & $0.277(4)$ & $1.079(4)$ & $0.919(4)$ & $0.05(1)$ \\
$\mathrm{H}(12 \mathrm{~B})$ & $2 i$ & $0.127(4)$ & $1.073(4)$ & $0.917(4)$ & $0.05(1)$ \\
$\mathrm{H}(14 \mathrm{~A})$ & $2 i$ & $0.255(5)$ & $1.296(5)$ & $0.521(4)$ & $0.07(1)$ \\
& & & & & \\
\hline
\end{tabular}

Table 2. Continued.

\begin{tabular}{llllll}
\hline Atom & Site & $x$ & $y$ & $z$ & $U_{\text {iso }}$ \\
\hline & & & & & \\
& $2 i$ & $0.392(5)$ & $1.203(4)$ & $0.494(4)$ & $0.06(1)$ \\
$\mathrm{H}(15 \mathrm{~A})$ & $2 i$ & $0.143(6)$ & $1.154(5)$ & $0.505(5)$ & $0.08(2)$ \\
$\mathrm{H}(15 \mathrm{~B})$ & $2 i$ & $0.256(5)$ & $1.181(5)$ & $0.389(5)$ & $0.07(1)$ \\
$\mathrm{H}(16 \mathrm{~A})$ & $2 i$ & $0.321(4)$ & $0.840(4)$ & $0.657(4)$ & $0.052(9)$ \\
$\mathrm{H}(16 \mathrm{~B})$ & $2 i$ & $0.167(5)$ & $0.939(4)$ & $0.684(4)$ & $0.06(1)$ \\
$\mathrm{H}(17 \mathrm{~A})$ & $2 i$ & 0.3165 & 0.9589 & 0.7912 & 0.079 \\
$\mathrm{H}(17 \mathrm{~B})$ & $2 i$ & 0.4376 & 0.9876 & 0.6624 & 0.079 \\
$\mathrm{H}(19)$ & $2 i$ & $0.262(7)$ & $1.076(6)$ & $0.267(6)$ & $0.11(2)$ \\
$\mathrm{H}(20)$ & $2 i$ & 0.215 & 0.964 & 0.1537 & 0.112 \\
$\mathrm{H}(21)$ & $2 i$ & 0.1367 & 0.7643 & 0.2591 & 0.092 \\
$\mathrm{H}(23)$ & $2 i$ & $0.200(5)$ & $0.771(4)$ & $0.583(5)$ & $0.07(1)$ \\
& & & & & \\
\hline
\end{tabular}

Table 3. Atomic coordinates and displacement parameters (in $\AA^{2}$ ).

\begin{tabular}{|c|c|c|c|c|c|c|c|c|c|c|}
\hline Atom & Site & $x$ & $y$ & $z$ & $U_{11}$ & $U_{22}$ & $U_{33}$ & $U_{12}$ & $U_{13}$ & $U_{23}$ \\
\hline $\mathrm{Cl}(1)$ & $2 i$ & $0.1139(1)$ & $0.5969(1)$ & $0.5139(1)$ & $0.1119(9)$ & $0.0694(7)$ & $0.0755(7)$ & $-0.0340(6)$ & $-0.0032(6)$ & $-0.0284(5)$ \\
\hline$C(1)$ & $2 i$ & $0.5535(6)$ & $1.5425(6)$ & $1.2971(6)$ & $0.081(3)$ & $0.121(4)$ & $0.117(4)$ & $-0.019(3)$ & $-0.036(3)$ & $-0.066(4)$ \\
\hline $\mathrm{C}(2)$ & $2 i$ & $0.4788(4)$ & $1.4268(4)$ & $1.1962(3)$ & $0.055(2)$ & $0.061(2)$ & $0.055(2)$ & $-0.014(2)$ & $-0.014(2)$ & $-0.018(2)$ \\
\hline$C(3)$ & $2 i$ & $0.3359(4)$ & $1.4877(4)$ & $1.2292(4)$ & $0.064(2)$ & $0.067(2)$ & $0.063(2)$ & $-0.010(2)$ & $-0.012(2)$ & $-0.035(2)$ \\
\hline$C(4)$ & $2 i$ & $0.2333(4)$ & $1.4596(4)$ & $1.1909(4)$ & $0.054(2)$ & $0.063(2)$ & $0.062(2)$ & $-0.009(2)$ & $-0.011(2)$ & $-0.030(2)$ \\
\hline $\mathrm{C}(5)$ & $2 i$ & $0.2712(4)$ & $1.3697(3)$ & $1.1204(3)$ & $0.055(2)$ & $0.053(2)$ & $0.044(2)$ & $-0.014(1)$ & $-0.012(1)$ & $-0.010(2)$ \\
\hline $\mathrm{C}(6)$ & $2 i$ & $0.4172(4)$ & $1.3106(4)$ & $1.0864(3)$ & $0.057(2)$ & $0.055(2)$ & $0.055(2)$ & $-0.010(2)$ & $-0.010(2)$ & $-0.020(2)$ \\
\hline $\mathrm{C}(7)$ & $2 i$ & $0.5182(4)$ & $1.3385(4)$ & $1.1226(4)$ & $0.052(2)$ & $0.061(2)$ & $0.064(2)$ & $-0.008(2)$ & $-0.013(2)$ & $-0.021(2)$ \\
\hline $\mathrm{C}(8)$ & $2 i$ & $0.1622(4)$ & $1.3317(3)$ & $1.0863(3)$ & $0.055(2)$ & $0.050(2)$ & $0.042(2)$ & $-0.012(1)$ & $-0.009(1)$ & $-0.012(1)$ \\
\hline$C(9)$ & $2 i$ & $0.0099(4)$ & $1.3736(4)$ & $1.1354(3)$ & $0.055(2)$ & $0.060(2)$ & $0.053(2)$ & $-0.014(2)$ & $-0.008(2)$ & $-0.020(2)$ \\
\hline $\mathrm{C}(10)$ & $2 i$ & $-0.0829(4)$ & $1.3286(4)$ & $1.1071(4)$ & $0.049(2)$ & $0.071(2)$ & $0.066(2)$ & $-0.016(2)$ & $-0.004(2)$ & $-0.023(2)$ \\
\hline$C(11)$ & $2 i$ & $-0.0332(4)$ & $1.2382(4)$ & $1.0295(3)$ & $0.062(2)$ & $0.058(2)$ & $0.052(2)$ & $-0.022(2)$ & $-0.016(2)$ & $-0.008(2)$ \\
\hline $\mathrm{C}(12)$ & $2 i$ & $0.1809(5)$ & $1.1240(4)$ & $0.9005(4)$ & $0.065(2)$ & $0.053(2)$ & $0.056(2)$ & $-0.016(2)$ & $-0.017(2)$ & $-0.018(2)$ \\
\hline$C(13)$ & $2 i$ & $0.1940(4)$ & $1.2066(3)$ & $0.7605(3)$ & $0.066(2)$ & $0.051(2)$ & $0.053(2)$ & $-0.018(2)$ & $-0.015(2)$ & $-0.015(1)$ \\
\hline$C(14)$ & $2 i$ & $0.2932(7)$ & $1.2067(5)$ & $0.5341(4)$ & $0.118(4)$ & $0.054(2)$ & $0.050(2)$ & $-0.025(2)$ & $0.000(2)$ & $-0.012(2)$ \\
\hline $\mathrm{C}(15)$ & $2 i$ & $0.2340(7)$ & $1.1419(4)$ & $0.4722(4)$ & $0.102(4)$ & $0.050(2)$ & $0.049(2)$ & $-0.011(2)$ & $-0.013(2)$ & $-0.005(2)$ \\
\hline$C(16)$ & $2 i$ & $0.2706(5)$ & $0.9328(4)$ & $0.6411(4)$ & $0.075(2)$ & $0.049(2)$ & $0.051(2)$ & $-0.008(2)$ & $-0.017(2)$ & $-0.009(2)$ \\
\hline $\mathrm{C}(17)$ & $2 i$ & $0.3343(5)$ & $0.9997(4)$ & $0.6986(4)$ & $0.078(2)$ & $0.060(2)$ & $0.058(2)$ & $-0.003(2)$ & $-0.020(2)$ & $-0.019(2)$ \\
\hline$C(18)$ & $2 i$ & $0.2484(4)$ & $0.9315(4)$ & $0.4363(3)$ & $0.068(2)$ & $0.053(2)$ & $0.049(2)$ & $-0.008(2)$ & $-0.012(2)$ & $-0.011(2)$ \\
\hline$C(19)$ & $2 i$ & $0.2522(6)$ & $0.9874(5)$ & $0.3049(4)$ & $0.127(4)$ & $0.060(2)$ & $0.056(2)$ & $-0.025(2)$ & $-0.031(2)$ & $-0.002(2)$ \\
\hline $\mathrm{C}(20)$ & $2 i$ & $0.2117(7)$ & $0.9246(5)$ & $0.2409(5)$ & $0.151(5)$ & $0.073(3)$ & $0.058(2)$ & $-0.019(3)$ & $-0.046(3)$ & $-0.006(2)$ \\
\hline $\mathrm{C}(21)$ & $2 i$ & $0.1663(6)$ & $0.8050(4)$ & $0.3023(5)$ & $0.100(3)$ & $0.062(2)$ & $0.072(3)$ & $-0.007(2)$ & $-0.036(2)$ & $-0.019(2)$ \\
\hline $\mathrm{C}(22)$ & $2 i$ & $0.1668(4)$ & $0.7495(4)$ & $0.4292(4)$ & $0.071(2)$ & $0.054(2)$ & $0.063(2)$ & $-0.010(2)$ & $-0.014(2)$ & $-0.017(2)$ \\
\hline $\mathrm{C}(23)$ & $2 i$ & $0.2059(4)$ & $0.8096(4)$ & $0.4970(4)$ & $0.076(2)$ & $0.056(2)$ & $0.045(2)$ & $-0.011(2)$ & $-0.011(2)$ & $-0.011(2)$ \\
\hline $\mathbf{N}(1)$ & $2 i$ & $0.2101(3)$ & $1.2530(3)$ & $1.0124(3)$ & $0.056(2)$ & $0.056(2)$ & $0.049(2)$ & $-0.013(1)$ & $-0.013(1)$ & $-0.013(1)$ \\
\hline$N(2)$ & $2 i$ & $0.1142(3)$ & $1.2088(3)$ & $0.9858(3)$ & $0.062(2)$ & $0.055(2)$ & $0.048(2)$ & $-0.017(1)$ & $-0.013(1)$ & $-0.016(1)$ \\
\hline$N(3)$ & $2 i$ & $0.2708(4)$ & $1.1415(3)$ & $0.6718(3)$ & $0.093(2)$ & $0.053(2)$ & $0.052(2)$ & $-0.016(2)$ & $-0.009(2)$ & $-0.015(1)$ \\
\hline$N(4)$ & $2 i$ & $0.2883(3)$ & $0.9964(3)$ & $0.5018(3)$ & $0.069(2)$ & $0.052(2)$ & $0.048(2)$ & $-0.014(1)$ & $-0.012(1)$ & $-0.009(1)$ \\
\hline$O(1)$ & $2 i$ & $0.5861(3)$ & $1.4460(3)$ & $1.2319(3)$ & $0.062(2)$ & $0.096(2)$ & $0.092(2)$ & $-0.011(1)$ & $-0.024(1)$ & $-0.048(2)$ \\
\hline$O(2)$ & $2 i$ & $-0.1115(3)$ & $1.1886(3)$ & $1.0036(3)$ & $0.071(2)$ & $0.081(2)$ & $0.087(2)$ & $-0.027(1)$ & $-0.021(1)$ & $-0.030(2)$ \\
\hline$O(3)$ & $2 i$ & $0.1379(3)$ & $1.3245(3)$ & $0.7322(3)$ & $0.097(2)$ & $0.052(2)$ & $0.060(2)$ & $-0.012(1)$ & $-0.018(1)$ & $-0.016(1)$ \\
\hline
\end{tabular}

Acknowledgment. The authors acknowledge the purchase of the CAD4 diffractometer under Grant DPT/TBAG1 of the Scientific and Technical Research Council of Turkey.

\section{References}

1. Gunesakaran, K.; Govindasamy, L.; Raj, S. S. S.; Velmurugan, D.; Karunakaran, S.; Kandaswamy, M.: 2,2'-Piperazin-1,4-diyl-dimethylenebis[6-(benzyliminomethyl)-4-methylphenol]. Acta Crystallogr. C52 (1996) 1027-1028.

2. Mairesse, P. G.; Boivin, J. C.; Thomas, D. J.; Bonte, J. ; Lesieur, D.: Structure de 1'(Ethynyl-1 cyclohexyloxy)-1[(Méthoxy-2 phényl)-4 pipérazinyl-1]-3 propanol-2-(RS). Acta Crystallogr. C43 (1987) 2128-2130
3. Blaton, N. M.; Peters, O. M.; Ranter, C. J.: 2-Methylthio-10,11dihydro-11-(4-methyl-piperazin-1-yl) dibenzo[b, $f$ thiepine Maleic Acid (Metitepine Maleate). Acta Crystallogr. C51 (1995) 777-780.

4. Moreau, S.; Metin, J.; Coudert, P.; Couquelet, J.: 5(2-Chlorobenzyl)6-methyl-3(2H)-pyridazinone. Acta Crystallogr. C51 (1995) 1834-1836.

5. Fronczek, F. R.; Oliver, M. A.; Gandour, R. D.: (E)-and(Z)-2,3-Bis(2methoxyphenyl)-2-butene, $\mathrm{C}_{18} \mathrm{H}_{20} \mathrm{O}_{2}$. Acta Crystallogr. C40 (1984) 504-506.

6. Fair, C. K. : MolEN. An Interactive Intelligent System for Crystal Structure Analysis. Enraf-Nonius, Delft, The Netherlands 1990.

7. Sheldrick, G. M.: SHELXS-97 Program for the Solution of Crystal Structures. University of Göttingen, Germany 1997.

8. Sheldrick, G. M.: SHELXL-97 Program for the Refinement of Crystal Structures. University of Göttingen, Germany 1997.

9. Johnson, C. K.: ORTEP-II. Report ORLN-5138. Oak Rigge National Laboratory, Tennessee, USA 1976. 